European journal of American studies

\title{
Legible Bodies and the Ghosts of American History: On Racialized Surveillance in Ubisoft's Watch Dogs Videogames
}

Sören Schoppmeier

\section{OpenEdition}

\section{Journals}

Electronic version

URL: https://journals.openedition.org/ejas/17324

DOI: 10.4000/ejas.17324

ISSN: 1991-9336

Publisher

European Association for American Studies

\section{Electronic reference}

Sören Schoppmeier, "Legible Bodies and the Ghosts of American History: On Racialized Surveillance in Ubisoft's Watch Dogs Videogames", European journal of American studies [Online], 16-3 | 2021, Online since 24 September 2021, connection on 24 September 2021. URL: http://journals.openedition.org/ ejas/17324 ; DOI: https://doi.org/10.4000/ejas.17324

This text was automatically generated on 24 September 2021.

Creative Commons License 


\title{
Legible Bodies and the Ghosts of American History: On Racialized Surveillance in Ubisoft's Watch Dogs Videogames
}

\author{
Sören Schoppmeier
}

\section{Introduction: All (Wo)men Are (not) Surveilled Equally}

1 The public reactions to both Edward Snowden's revelations about the NSA's PRISM program (cf. Greenwald) and to the growing comprehension of the extent and pervasiveness of online tracking by "surveillance capitalists" like Google and Facebook (Zuboff 8) share a conspicuous element: what appears to be most shocking to the public is the fact that everyone is affected by digital surveillance. The outrage, of course, is justified; everyone has a right to privacy, even as this right is steadily eroded in an always-online world. Yet the sentiment also reveals a peculiar perception of extant surveillance practices. Fixating on the ubiquity and universality of contemporary digital surveillance implies that, perhaps, things would not be as bad if only some people were surveilled, people other than oneself. Shock at the sudden realization of being surveilled without a proper cause betrays a certain privilege; surveillance here is perceived as something not only unwarranted but unexpected, even unfamiliar.

2 The centrality of the factor of experience exposes the historical, cultural, and political dimensions of surveillance, particularly in the United States. Despite being ubiquitous and comprehensive today, surveillance is by no means universal. "Surveillance," as Simone Browne reminds us, "is nothing new to black folks. It is the fact of antiblackness" (10). A similar argument can be made about many groups and individuals outside of the dominant White, Christian, heterosexual norm who have long 
been subjected to different forms of state surveillance, including BIPOC (Black, Indigenous and people of color), Muslim Americans, and LGBTQIA people.

In this article, I analyze Ubisoft's Watch Dogs and Watch Dogs 2 (henceforth referred to as WD and WD2), ${ }^{1}$ two AAA open-world videogames, ${ }^{2}$ to show how the games reproduce the racialized (ine)qualities of surveillance practices in the United States. Previous scholarship on the series has already shed light on the videogames' representation of contemporary surveillance regimes. This article complements that research with a special concentration on Watch Dogs' racial/racist dimension. David J. Leonard's article "Virtual Anti-Racism: Pleasure, Catharsis, and Hope in Mafia III and Watch Dogs 2" is a precursor of this angle, but it only considers WD2 and focuses exclusively on the game's narrative layer, its theme of resistance, and the few examples where systemic racism is explicitly addressed. Delving deeper into matters of racialized surveillance and drawing especially on recent research at the intersections of STS (science and technology studies) and CRT (critical race theory) by scholars like Ruha Benjamin and Simone Browne, I scrutinize aspects of Watch Dogs' fictional world, game mechanics, plot, and visual and procedural representation. I argue that the representation of surveillance in the first two Watch Dogs titles works to conceal the uneven application and effects of surveillance in the real world. At the same time, the colorblindness governing the gameworlds throws into relief the racializing nature of surveillance as expressed through some of the characters. Here surveillance presents itself as "racializing surveillance-when enactments of surveillance reify boundaries along racial lines, thereby reifying race, and where the outcome of this is often discriminatory and violent treatment" (Browne 8). The Watch Dogs videogames both erase the realities of racializing surveillance in their conceptualization and simulation of a contemporary American "surveillance society" (Marx 5) and prominently feature characters who embody the painful histories and present of racializing surveillance in the United States. These two opposing representations, I argue, ultimately reproduce the racializing logics of contemporary digital surveillance as well as its lineage in American history as both whiteness and Blackness organize the operation of surveillance in the videogames.

4 My argument begins with an analysis of the Watch Dogs franchise's gameworlds as expressed through the depiction of digital profiling, exposing a colorblindness that works to erase racializing differences within a comprehensive surveillance regime. Then I turn to the question of biometrics, connecting the reliance on facial recognition technology in the series' "playable representations" (Murray 25) and one of WD's subplots with the origins of biometrics in the transatlantic slave trade. The third section concerns the videogames' depiction of predictive policing technology, which is analyzed against the background and racialized underpinnings of similar practices utilized by police departments across the United States. Finally, the conclusion synthesizes the preceding insights in order to illuminate the cultural work of the Watch Dogs videogames vis-à-vis the past, present, and future of racialized/racializing surveillance in the United States. 


\section{All-Seeing but Colorblind: Digital Profiling and the World of Watch Dogs}

5 Set in contemporary Chicago and San Francisco, respectively, WD (2014) and WD2 (2016) are gamic works of speculative fiction that depict American metropoles subtly but substantially transformed through extrapolations of technological developments already existent in the real world. "Speculative fiction," Gerald R. Lucas writes, "will often answer an implied 'What if?' question that posits an alternative reality as its primary narrative drive. The distorted or altered reality explicitly propels the narrative while implicitly challenging quotidian assumptions of reality and those forces that comprise it-history, science, technology, politics, and metaphysics" (4). The questions at the heart of Watch Dogs is: What if the smart city, ubiquitous surveillance of public spaces, and digital profiling based on tracking online behavior became more than comprehensive? What if they also became converged into one unified system governing an entire city, in a public-private cooperation fusing the disparate activities of corporate actors and municipal authorities?

6 The cities in Watch Dogs are run on a unified operating system called ctOS, which connects physical infrastructure, citizen data, and law enforcement practices. Developed and run by Blume, a corporation reminiscent of real-world Silicon Valley powerhouses, ctOS ostensibly secures the smooth functioning of Chicago and San Francisco while, by extension, also providing online access to each and every element that the city comprises, from traffic lights to its residents. Surveillance cameras covering nearly every spot of the cities are connected to facial recognition software, which is linked to a comprehensive database of all residents. In Watch Dogs, then, being in public is tantamount to being known and locatable; ctOS works as an all-seeing eye.

7 Consequently, access to ctOS means access to the residents' digital profiles. As the protagonists in WD and WD2-Aiden Pearce and Marcus Holloway, respectively-are exceptional hackers, players are presented with a short profile of passers-by simply by using the profiler app on the player characters' smartphones with the push of a button. From transparent cities to transparent citizens, all seems to be knowable and governable to those in control of ctOS.

While several layers of this dynamic are pertinent here-and many are addressed in the videogames' narratives-I will focus specifically on the digital citizen profiles. Although a more detailed profile is implied and referenced repeatedly, and even briefly displayed in Marcus's case early in WD2, players only see an abridged version. It includes a photo, the character's name, age, occupation, income, and a supposedly notable fact(oid) about them. The latter ranges from "frequents racist blogs" to "victim of childhood abuse" and everything in between. The "prostitute" who is "[r]esearching tech startups" in WD2 is one of an indefinite number of examples (Fig. 1). 


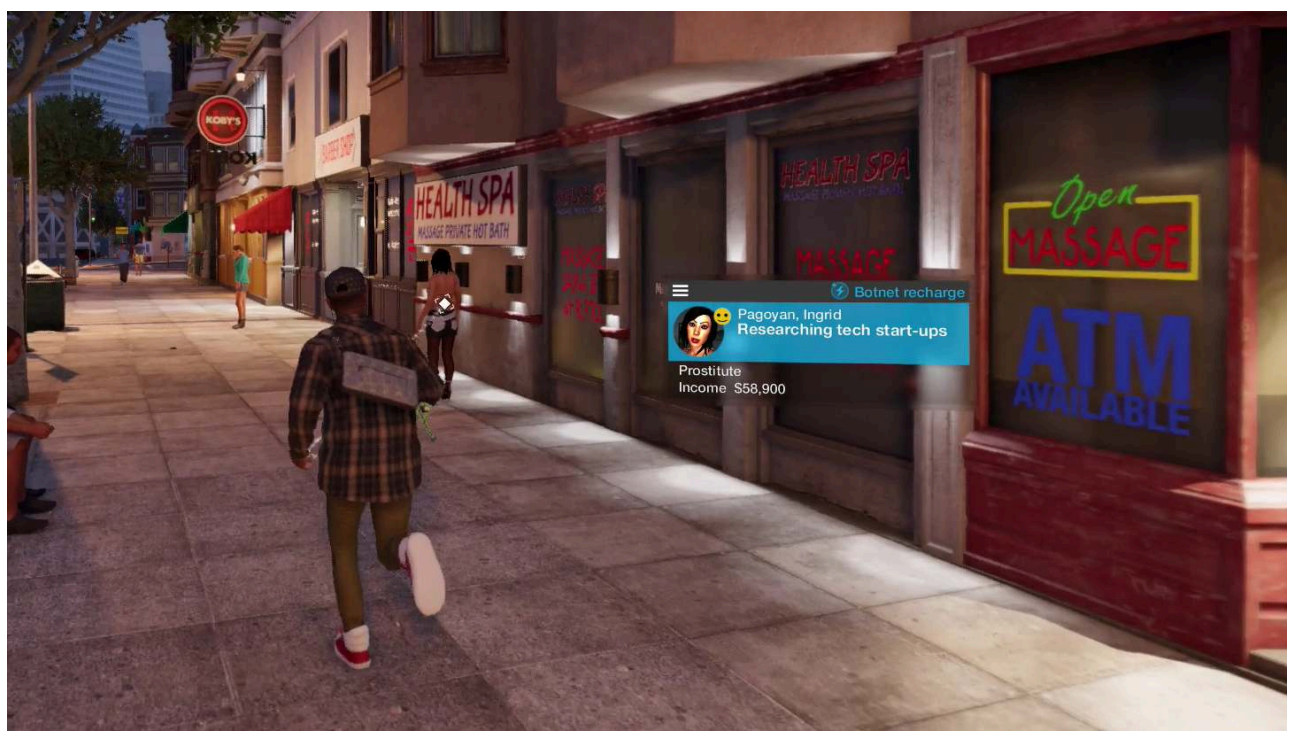

Fig. 1: Scanning an $\mathrm{NPC}^{3}$ profile (Watch Dogs 2, screenshot taken by author).

9 Since these profiles, along with snippets of phone calls or text messages, are all that players can find out about NPCs irrelevant to the plot, they function as proxies representing those people. This is not unlike some real-world practices of big data analytics, in which "[t]he inclination is to replace people with data trails" (O'Neil 48). This "runaway data" (Pasquale 21) amounts to what Shoshana Zuboff calls "behavioral surplus" (75) and describes as the "[r]aw materials" (74) for surveillance capitalism's immensely profitable "prediction products" (96). The boundaries between private and public sector surveillance here are increasingly blurred (Pasquale 21, 42-48), and digital personal profiles are a decisive element of the surveillance-based technological and social transformations of our time.

10 Concerning questions of racialized surveillance, it is necessary to consider Watch Dogs profiles from a procedural as well as representational perspective. According to the developers, not only are no two profiles the same (Sinha), allegedly they are also randomly generated for NPCs unrelated to the videogames' missions and not handcrafted with all segments of a profile carefully matched with each other (Imtiaz). Instead, an algorithm procedurally generates a profile by arbitrarily picking and combining values from a database. Nonetheless, Watch Dogs' profiles are far from being neutral window dressing. Discussing a now infamous video of WD gameplay called "Making the World a Better Place," Jennifer R. Whitson and Bart Simon stress that

there are implicit values and politics associated with the database list of deviant 'personality' features that players may choose to act on. Many of the categories are 'common sense' or even comedic notions of deviance and otherness presumably from the perspective of the white heterosexual male that is the protagonist of the story. What we get with this intriguing system for making decisions in the gameworld is not a celebration of difference or plurality but a mechanism for defining deviance and otherness against some undefined norm. What is also clear is that there is a finite set of 'otherness' categories (mostly likely limited by both technical constraints and developers' imaginations) which must be assigned to all characters in the game (except of the player). (315)

Players hence witness an obsession with some notion of social deviance, presumably from a "white heterosexual male" norm, while the profiles simultaneously appear to operate as an algorithmic equalizer. NPCs are overwhelmingly represented as deviant 
in some way, meaning that everyone is deviant in this world. As none of the characters' features relate to any other in any discernibly static way, removing explicit discrimination from the equation, the approach is implicitly colorblind. In Watch Dogs, lo and behold, all (wo)men are created equal(ly). Yet this is exactly why the videogames' representation of surveillance and digital profiling is troubling.

The depiction of NPC profiles resembles their real-world counterparts on a conceptual level, especially in their functioning as black boxes-diegetically, there is no way to assess how the profiles were assembled. "The term "black box," Frank Pasquale writes, "is a useful metaphor ... given its dual meaning. It can refer to a recording device, like the data-monitoring systems in planes, trains, and cars. Or it can mean a system whose workings are mysterious; we can observe its inputs and outputs, but we cannot tell how one becomes the other" (3). Nonetheless, fixating on the ubiquity, comprehensiveness, and universalism of contemporary digital surveillance-alas, everyone is affected!obscures another layer that originates in histories older than computation and that is deeply ingrained in the social structures and technological designs in which these practices are embedded. Benjamin cautions us that "a universalizing lens may actually hide many of the dangers of discriminatory design, because in many ways Black people already live in the future" (32). That is, some people, particularly BIPOC, have been subjected to surveillance and profiling long before computational algorithms took over. By the time surveillance becomes universal-read: when it also affects white Americans on a large scale and across classes-it has long been applied to marginalized groups. What is novel and shocking to some has been an expected fact of life for others. The Watch Dogs videogames, then, distort the reality of surveillance in the United States. As profiles are generated in a random manner, they do not account for the ways in which real-life profiles based on digital surveillance are frequently assembled along fault lines of difference that are deeply entrenched in discriminatory social and cultural structures and practices that have evolved over generations.

The world of Watch Dogs appears colorblind: its character generation model does not differentiate between people based on race, but it also inadvertently ignores the unequal effects a comprehensive surveillance regime as depicted here has in a real world where race still is a determinant of life experiences. Some missions, particularly in WD2, address precisely these points on a narrative level. Especially Marcus's case is salient. Early in the game, he is introduced as someone frequently targeted-read: racially profiled-and falsely accused by authorities through ctOS. Yet this perspective is conspicuously absent on a systemic, simulational level. If there is racial profiling through ctOS, players would not know by simply walking the streets and paying attention to their surroundings; for all that has happened to Marcus, not even he ever gets targeted outside of scripted missions.

By not implementing the "white gaze" ("Toni Morrison")-a dominant, racialized viewing position that uncritically assumes itself as an unmarked norm ${ }^{5}$-that permeates surveillance technology in the fictional system depicted in the videogames, Watch Dogs actually reproduces a perspective suffused with "[t]he invisible power of Whiteness" (Benjamin 29). Its representation of digital profiling remains untouched by issues of race-a privilege based on "a location of structural advantage" and upheld by "a set of cultural practices that are usually unmarked and unnamed" (Frankenberg 1). "Database design," Benjamin asserts, is "an exercise in worldbuilding," a normative process in which programmers are in a position to project their world views-a process 
that all too often reproduces the technology of race" (78). Race often creeps into and informs technological design unless explicitly countered. It is imperative to "consider race as itself a technology, as a means to sort, organize, and design a social structure" (Benjamin 91), which also illustrates why questions of race become urgent with regard to material technologies that execute procedures devised precisely "to sort, organize, and design."

This particular representation of surveillance in Watch Dogs constitutes a form of racialized, but also racializing, surveillance precisely because it operates from a position of whiteness. The unmarked perspective itself must be viewed as racialized since it works in a manner that racializes both subject and object-that is, the player and the NPCs. This point is most obvious in WD, whose protagonist is white and well aware of his scopic power. Early in the game, as players first use the profiler app, Aiden remarks, "this city's full of distractions, and they can't hide from me." His whiteness comes full circle when considering that Aiden erased his own profile by hacking ctoS, which not only exempts him from being surveilled but renders him unmarked and invisible. Even in WD2, where the protagonist is Black and where representational diversity is exceptionally inclusive for a AAA videogame, the player's gaze operates from a position of whiteness precisely because neither their nor Marcus' identity affect the system itself. There is no essential difference between WD and WD2 here; although their approaches to representation differ greatly, the system underlying the simulation remains the same.

The white perspective's persistence in Watch Dogs is particularly prominent when the ease of using the profiler regularly prompts players to take certain actions over others. The ready availability of NPC profiles "turns casual inattentiveness into focused watchfulness in which every encounter becomes a matter of concern" (Whitson and Simon 315), eventually impacting players' gameplay decisions, such as which NPC to rob or even kill. Although the procedurally-generated profiles are randomized and do not insinuate any correlations between race and the information displayed, the player's viewing position aligns with a scopic regime defined by whiteness-constantly watching, categorizing, and judging Others while never subjected to the same kind of mechanism. If "domination and surveillance typically go hand in hand with 'the pleasure of looking"' (Benjamin 110), the gamic pleasures of scanning NPC profiles in Watch Dogs betray a similar power dynamic.

Digital profiling in these videogames, then, constitutes a form of racializing surveillance since it erases the disproportionate and unequal subjection of BIPOC to ubiquitous surveillance online and offline in the real world while simultaneously replicating a watching position defined by whiteness for the player. "It is tempting," Benjamin reminds her readers, "to point out the smart recording devices we carry around in our pockets and exclaim that "we are all caught inside the digital dragnet!" (111). But even if we are, "we do not all experience the dangers of exposure in equal measure" (Benjamin 111). For a videogame franchise aspiring to produce cautionary works of speculative fiction, this raises the problem that the projected world disregards the racialized implications of the lived reality on which it is based. It essentially reproduces a discourse of universalism concerning twenty-first-century surveillance practices that negates long-standing and ongoing struggles against surveillance by groups outside of the dominant white norm. The following section delves deeper into 
this history by turning to the technological method behind Watch Dogs' profiler: biometrics.

\section{Reading the (Black) Body: Biometrics, Racializing Surveillance, and the White Ancestors of ctOS} history of slavery, and the racializing (and racist) practices of both historical and contemporary surveillance, Browne provides a powerful account of "the facticity of surveillance in black life" (7). Her intervention urges us to be wary of the racial politics of any account or representation of surveillance (9).

The profiler's functioning in Watch Dogs relies on the city-wide application of facialrecognition software operating surveillance cameras, connected to the database of residents' profiles. Facial recognition technology is one branch of a set of identification techniques generally referred to as biometrics. Browne contends that "biometrics is a technology of measuring the living body. The application of this technology is in the verification, identification, and automation practices that enable the body to function as evidence" (109). The connection between the body and information technologyindeed, the body as information technology-is crucial here. Browne traces the history of biometrics back to the transatlantic slave trade to demonstrate "that this history is in close alignment with the commodification of blackness" (128). She concludes:

Current biometric technologies and slave branding, of course, are not one and the same; however, when we think of our contemporary moment when 'suspect' citizens, trusted travelers, prisoners, welfare recipients, and others are having their bodies informationalized by way of biometric surveillance, sometimes voluntarily and sometimes without consent or awareness, and then stored in large-scale, automated databases, some managed by the state and some owned by private interests, we can find histories of these accountings of the body in, for example, the inventory that is the Book of Negroes, slave ship manifests that served maritime insurance purposes, banks that issued insurance policies to slave owners against the loss of enslaved laborers, and branding as a technology of tracking blackness that sought to make certain bodies legible as property. (128)

Considering slave branding as one of the origins of biometrics, this horrendous practice makes an uncanny appearance in WD's narrative when Aiden tracks down a human trafficking ring over a series of missions. During one of these, "A Risky Bid," players infiltrate a secret auction of women-unmistakably sex slaves-presented on a stage in front of potential bidders. While the women's ethnicities vary, the visual similarity to the auctions of African slaves on the American continent during the $17^{\text {th }}, 18^{\text {th }}$, and $19^{\text {th }}$ centuries is striking. The history and horror of the transatlantic slave trade and American slavery acquire a haunting presence in one of WD's bleakest scenes as their visual archive collapses into a videogame representation of contemporary human trafficking enabled by digital technology.

Prophetically called "Stare into the Abyss," the preceding mission features an even more uncanny detail. Gathering intelligence about the human trafficking ring, Aiden learns that he may find information in a clandestine fetish club. He gains access by posing as Nicholas Crispin, a rich expatriate with a history of brutalizing and killing women for sexual pleasure. As Aiden enters the club, he finds that the club's owner has prepared a "gift" for Crispin, which also includes an invitation to the auction. The 
"gift" is a young African American woman called Poppy. Almost entirely naked, she has been made to wait in a room for Crispin so that he be able to do to her whatever pleases him. Her appearance, however, stands out for a different reason. Poppy's head is shaved clean and she wears a number of visible metal piercings: a nose ring attached by two chains to an ear ring, which is connected to another ear ring by several other chains-a truly evocative appearance. Poppy, having heard about Crispin's violent abuse, attacks Aiden as he enters the room, but he can fend her off and explain that he is not Crispin and that he wants to help her. Just before her ambush, Aiden catches a glimpse of what seems to be a small tattoo on the woman's neck. When Aiden infiltrates the auction and meets Poppy again, he asks her about the tattoo. She responds, "It's a tracking device beneath the skin. It's all so very efficient for business."

Within two short cutscenes, a specter appears. It is the specter of the brutal history of the transatlantic slave trade and American slavery, and of their instrumental role in the development of biometrics. In WD's speculative 2014 Chicago, players encounter a young African American woman being held as a slave to white men. The woman's body is marked and made legible twofold. Diegetically, Poppy's tattoo marks her as someone else's property, enabling that someone to track her everywhere she goes. The visual cues-her skin, facial features, shaved head, piercings, and chains-also mark her as more than an African American woman. Her bodily appearance urges players to read her as a revenant of American slavery; Poppy's body is legible to the player beyond the fiction of the videogame. This may not be the result of the developers' intentions. More likely, Poppy's character design constitutes yet another example of the common reproduction of long-standing and persistent stereotypes that frequently exoticize African American women and mark them as the Other in American culture, indeed the negative upon which white male supremacy rests. With its gratuitous depiction of vulnerable, naked women, this example also demonstrates how the white gaze, in the game as well as in past and present surveillance regimes, is closely coupled with a heterosexual male gaze that both controls and takes pleasure in watching female bodies (cf. Mulvey). ${ }^{6}$

This racialized and racializing operation of biometrics goes beyond the diegetic level as it invites WD players, too, to decode the information encoded in Poppy's body through something akin to "a technology of tracking blackness that sought to make certain bodies legible as property" (Browne 128). In these moments, the violent history of American slavery and the commodification of Black bodies returns like an apparition in the figure of Poppy-in her Black female body, specifically-and converges with twenty-first-century biometric surveillance technology. Alas, WD does not engage the symbolic significance of this extraordinary scene any further, and instead, Watch Dogs' operation from a position of whiteness becomes apparent. Aiden, true to the racist trope of the white savior, simply scrambles the signal of the tracking device beneath Poppy's skin with his phone, and she is free to flee (Fig. 2). 


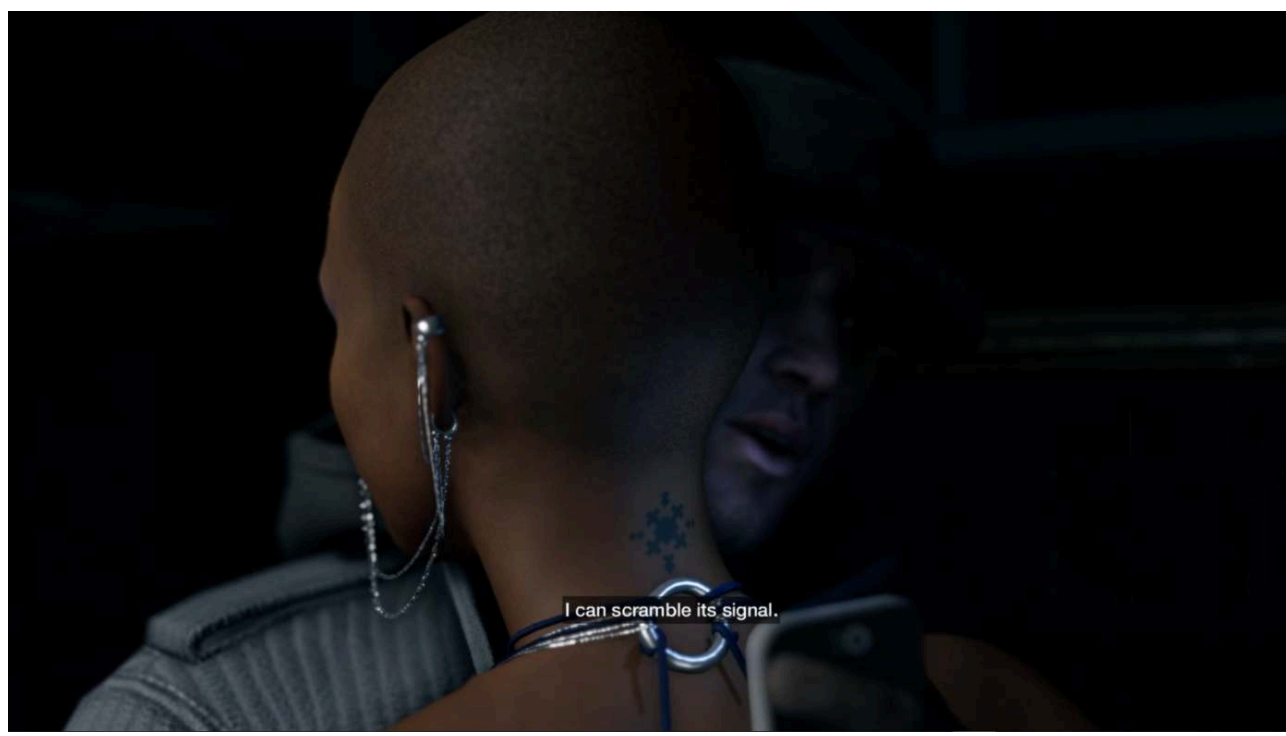

Fig. 2: Aiden scrambles the signal of Poppy's tracking device (Watch Dogs, screenshot taken by author).

Here, Watch Dogs' colorblindness shows itself as ignorance, disavowing the past suffering of, and ongoing discrimination against, BIPOC in the United States, which began with American slavery and settler colonialism and which continues under "the New Jim Code," which Benjamin defines as "the employment of new technologies that reflect and reproduce existing inequities but that are promoted and perceived as more objective or progressive than the discriminatory systems of a previous era" (5-6). Biometric technology, including current facial recognition technology, continues to be an important site of the entanglements of racialized surveillance, technological innovation, and discrimination. Its depiction in Watch Dogs, however, veers closer to a vantage point akin to the privilege bestowed by whiteness, which is one way in which (the representation of) surveillance practices can work as racializing. Real-life applications of facial recognition technology in the United States, especially in law enforcement, often rely on databases containing disproportionate numbers of BIPOC, especially African Americans, while the technology itself works rather poorly with faces of darker skin complexion (Benjamin 112). In contrast, Watch Dogs' ctOS seems to treat every resident the same, which portrays such technologies in a more benevolent way than they deserve. ${ }^{7}$ A similar approach can be observed with regard to predictive policing practices, whose representation in Watch Dogs is discussed next.

\section{From the Infallible to the Invisible: Whitewashing Predictive Policing}

Aiden Pearce is not merely a hacker; he is also a vigilante who acts on crime and misbehavior in a series of optional missions called Crime Detection Events, which "effectively results in enlisting the player (via Aiden) to enforce ctOS's legal order" (Ng and Macdonald 182). While roaming the streets of Chicago, WD players occasionally get alerted about impending crimes about to happen in their vicinity. These have been predicted by ctOS before they actually occur. If the player decides to act on an alert, they have to find the potential victim first, which is achieved by profiling nearby NPCs. Once the victim is identified, Aiden has to remain unseen lest he prematurely scare off 
the perpetrator. As the player surveils the potential victim, a continuously filling percentage bar in their profile indicates the growing impendence of the crime (Fig. 3). Only once absolutely imminent, when the perpetrator approaches the victim, players should interfere and neutralize the criminal. The altercation's outcome also affects the protagonist's public reputation, the way other NPCs react to him and his actions; for example, it impacts whether or not NPCs call the police on Aiden.

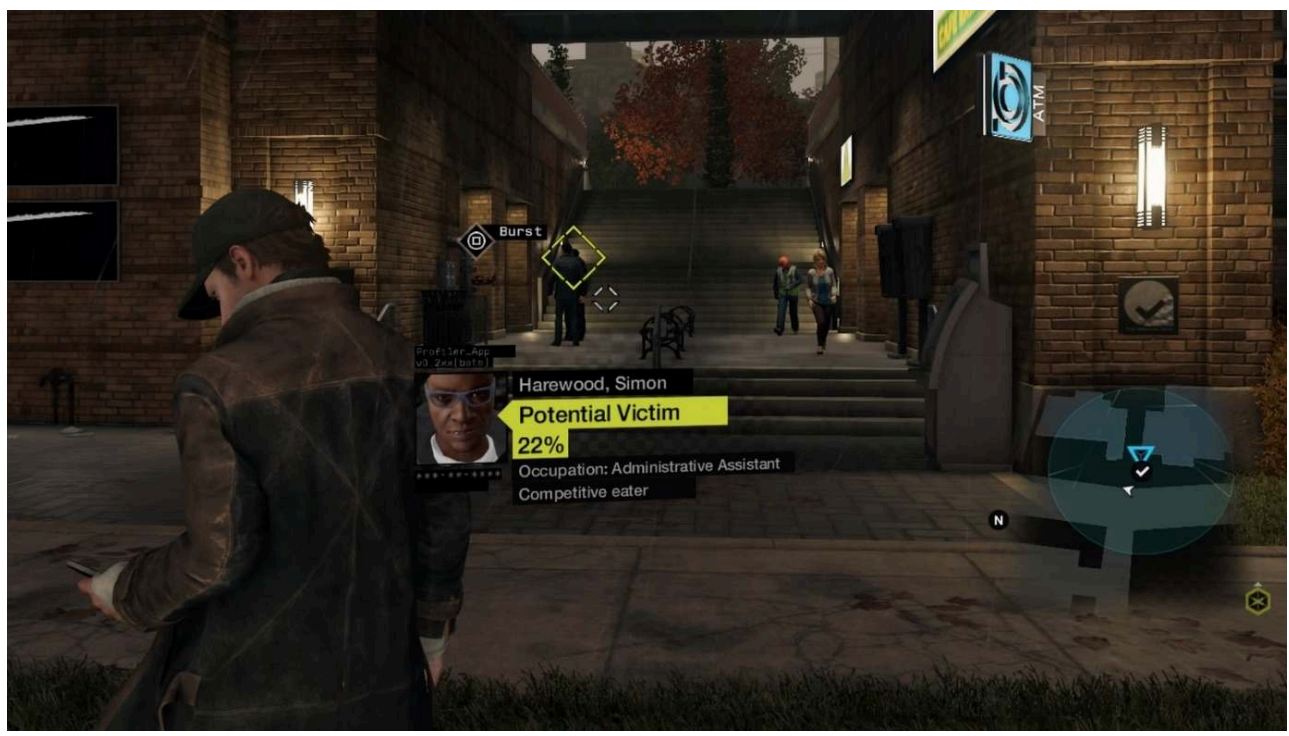

Fig. 3: A Crime Detection Event (Watch Dogs, screenshot taken by author)

Before analyzing these Crime Detection Events, which do not reappear in WD2, ${ }^{8}$ for their underlying logics and political implications, I shall address Ubisoft's inspirations. WD's fictional forebears comprise science-fiction classics like Philip K. Dick's short story "The Minority Report" and more recent works like Jonathan Nolan's television series Person of Interest. Beyond fiction, however, another major inspiration for the videogame is the reality of twenty-first-century law enforcement in the United States. Predictive policing is real and already applied in cities like Chicago, where WD is set, and Los Angeles. In the words of Andrew Guthrie Ferguson: "This is the future. This is the present. This is the beginning of big data policing" (2). With the help of big data analytics and machine-learning algorithms, "[p]olice can identify the street corner most likely to see the next car theft or the people most likely to be shot" (Ferguson 3). The two examples mentioned here embody the two main approaches found in current predictive policing technologies: place-based policing and person-based policing. The former refers to the prediction of hot spots where crime is likely to occur at a specific time, while the latter concerns the identification of individuals who, due to their personal networks and behavior, will probably be involved in criminal activity. Both approaches rely on large amounts of historical crime data that is automatically transformed into forecasts through analysis, interpretation, and extrapolation by machine-learning algorithms.

Although ostensibly objective, the very design of these technologies produces instances of discrimination as it reproduces racist patterns of the past. For example, non-white communities, especially African Americans, have historically been disproportionately targeted by law enforcement. If this biased data is used to train machine-learning algorithms, they will likely reproduce the bias even if race is not a factor in the algorithms themselves (Ferguson 47-52). Especially place-based predictive policing 
"runs the risk of creating its own self-fulfilling prediction" here, as Ferguson reminds his readers (74). Pasquale draws a similar conclusion when he reasons that "if police focus their efforts on minority communities, more minorities may end up with criminal records, regardless of whether minorities generally commit more crimes" (38). Rather than reducing bias in policing, then, such procedures may reinforce established racist patterns while cloaking them in a veneer of algorithmic neutrality. As predictive policing focuses on particular kinds of crime, which correlate with a particular class, which correlates with particular places, which correlate with non-white minorities (cf. Ferguson 75; Benjamin 35), it is ill-equipped to reduce racist bias and discrimination. If anything, predictive policing has so far shown to result in the same focus on BIPOC, especially African Americans, as its forebears in traditional, analog policing (Ferguson 47-52, 73-79). Predictive policing in the United States, therefore, is a prime example of ongoing practices of racialized surveillance with new, algorithmic tools, in which "the output of Jim Crow policies" becomes "the input of New Jim Code practices" (Benjamin 147).

The procedural and representational logics of WD's Crime Detection Events must be understood against the background of such real-world law enforcement techniques, not least because WD is set in a city where these are presently used. As presented to players, the crime prediction algorithm appears to be person-based and restricted to one type of crime. All instances exclusively feature an individual physically assaulting another individual, both of which appear random concerning markers of identity. Like its real-world counterparts, the system is black-boxed, so players never get to ascertain the data and mechanism behind the flagging of suspects; at best, they receive a suggestive sample of a text message or phone call indicating imminent danger.

The process of how the Crime Detection Events play out is significant. First, players have to engage in the surveillance of NPCs in their nearby surroundings. Using the profiler app to scan the people around Aiden, one must quickly identify the potential victim. This mirrors the large-scale, real-world surveillance of innocent and unsuspicious citizens to identify wanted suspects among them, which often has a racialized dimension when a particular group is targeted because of superficial, assumed similarities between them and the suspect, often boiling down to visual markers like skin tone, hair and eye color, hairstyle, and dress. This racialized dimension is conspicuously absent in WD's iteration of the practice. Since players do not know who they are looking for, they must consider everyone as potentially involved; racial profiling would be futile. Simultaneously, the person-based system also shows a dimension of place. The initial information only suggests that a crime will happen nearby, which creates a small geographical zone on which to focus one's surveillance. In the videogame, there is no indication that the location has been determined by historical data informed by racist structures; it purely relates to the likely whereabouts of the targets in question. The policing of places in WD as an intermediate step to policing people, then, appears to be detached from the histories of those places themselves and of the people who inhabit them-quite unlike the procedures applied by police across the United States.

Another remarkable aspect of the Crime Detection Events concerns the accuracy of ctOS' system. If players ignore an alert, they have no way of finding out whether the crime was eventually committed. "Such," writes Ferguson, "is the nature of prediction: It could be the future. Or not" (64). If WD players act on the prediction, however, the 
alert always proves correct; there are no false positives in ctOS' prediction algorithm. The only ways to fail these missions are scaring off the perpetrator too early and failing to intervene in time (cf. $\mathrm{Ng}$ and MacDonald 182). The logic behind this design is that humans make mistakes while computers do not, which is easily disproven and yet frequently serves as a justification for relying more on automation through complex computational algorithms. The circumstance that algorithms often carry over the biases of their creators and that they are prone to errors themselves, besides relying on data often already infested with error, becomes a concern only after the fact (cf. Ferguson 50-53). With its infallible algorithms, then, ctOS is closer to the utopia presented by predictive policing marketers than to the disillusioning reality of predictive policing in practice.

By rendering predictive policing universal, infallible, and colorblind, WD erases the lived realities of poor and predominately African American communities in the United States who have been the major target of policing for centuries. Operating from a privileged perspective of whiteness, WD's representation of predictive policing foregrounds the merits that have been promised but not realized, and it omits any indication of potential disparities in how different types of people are handled by the system. Surveillance is racialized here because it is whitewashed. As race is omitted as a discernible factor in WD's depiction of predictive policing, the videogame is all the more haunted by the racist practices it ignores.

\section{Conclusion: Legible Bodies and the Ghosts of American History}

For BIPOC in the United States, surveillance by the very country they live in, and whose civil rights most of them should be guaranteed by virtue of their citizenship, has long been an everyday reality. Any consideration of surveillance, whether its real-world application or fictional representations in popular culture, must deal with its racist and anti-Black history, particularly in a US context. Failing to account for the racialized and racializing nature of surveillance runs the risk of erasing the unequal experiences of different groups subjected to it in varying degrees and under disparate circumstances. That twenty-first-century biometrics make all bodies legible must not obscure the fact that non-white bodies have been made legible to those in power long before the advent of algorithmic surveillance. Technology works as an amplifier here: less than creating new practices, it facilitates established ones.

As Benjamin stresses, this "is about how historical processes make the present possible; it is about the continuity between Jim Crow and the New Jim Code" (147). These continuities are the ghosts of American history that haunt not only contemporary digital surveillance but also its representations in popular culture. These specters are haunting the Watch Dogs videogames precisely because they have largely been rendered invisible. For all the dystopian ambitions the franchise may have-even if it does not ultimately succeed in producing a dystopia (Hennig 8)-its speculation on the near future operates from a position of relative privilege as it erases many realities to which marginalized people are already subjected.

There is no doubt that WD2's display of diversity on the level of characters, narrative, and themes are an exceptional achievement in a AAA videogame market still 
overwhelmingly privileging white, male perspectives-particularly as its protagonist is an African American man who has fallen victim to racial profiling (cf. Leonard 3-4). ${ }^{9}$ Foregrounding the perspective of whiteness in the design of Watch Dogs' world and its procedures does not devalue the significance of Marcus as a protagonist and the importance of his experiences-at one point, for example, a camera fails to recognize him because his "face is too dark"-especially for players who may belong to marginalized communities themselves (cf. Leonard 3-7, 13-15). It rather highlights the colorblindness that exists next to a representational layer championing diversity and anti-racist politics on the surface and that runs much deeper in the simulation underneath. Paradoxically, racialized surveillance is both overlooked in the procedural design of Watch Dogs-even if it occasionally appears on the narrative level-and reproduced as surveillance by the player who always operates from a privileged position, ${ }^{10}$ which aligns with the structural advantage of whiteness. As Soraya Murray aptly summarizes, "[e]ven the refusal to engage with identity is a privilege that only a particular segment of the population is able to sustain, through their perceived normativity" (46).

Addressing the "cruel optimism" of videogames by drawing on the work of Lauren Berlant, Lisa Nakamura writes that

[g]ames make vividly visible what transformation might look like. The strict procedurality of games both satisfies and ignites desires for fairness in the context of a US culture that is patently unfair, particularly for racial minorities and women. The attachment to games can be a cruel one for all players, but especially for those who are subject to even more unfair proceduralities and forms of systematic discrimination in real life. (248)

In the context of contemporary digital surveillance, fixating on universality can have the effect of ignoring or even delegitimizing that same regime's ongoing unequal effects on different (groups of) people, which ultimately perpetuates discrimination. It is evident that popular culture is one channel through which surveillance technology becomes known to and is normalized by the general population (Browne 121). Videogames are a form of popular culture that not only depicts such technologies but allows players to engage with them on a simulational, systemic level.

Understanding how surveillance and whiteness work together in the world of Watch Dogs sheds light on the currency popular notions of colorblind technology have even within critical discourses on surveillance. Whereas the scholars invoked throughout my argument provide theoretical considerations of the racial dimensions of computational technology and accounts of racializing and racist institutional procedures, this article supplements a correspondent perspective of representational practice and popular imaginations. Representations like those in the Watch Dogs videogames perform the cultural work of enabling ongoing applications of racializing surveillance precisely because the shock factor of their simulations relies on supposedly indiscriminate surveillance, which negates questions of difference. Attending to such conflicted representations allows us as scholars of American studies to trace the operation of whiteness in practice and produce critical counternarratives to dominant accounts of surveillance, as demanded by scholars like Browne (8-9).

Urging the field of "game studies to take seriously the ways in which games and gaming culture are indelibly marked by the reanimation of white supremacy," TreaAndrea Russworm asks: "Specifically, what might it mean for the field of game studies to make the persistence of white supremacy central to how we play, write about, and teach 
video games?" (75). One answer to this question is to attend to the gaps and silences in videogame representations. "Anti-Blackness is no glitch" (Benjamin 82); it is a feature of surveillance technologies in the United States. Fixating on the black box of surveillance on its own terms can easily obscure how quickly and how frequently it turns into an "anti-Black box" (Benjamin 35). Surveillance in the United States has always been racialized and racializing. Representations that do not take this into account are ill-equipped to offer effective critiques of comprehensive surveillance regimes like that depicted in Watch Dogs. At worst, they may render invisible the struggles of those who are most exposed.

\section{BIBLIOGRAPHY}

Benjamin, Ruha. Race after Technology: Abolitionist Tools for the New Jim Code. Polity, 2019.

Browne, Simone. Dark Matters: On the Surveillance of Blackness. Duke UP, 2015.

D, Tanja. "Racism in Watch Dogs 2 Is Subtle, and that's How It Won Me Over." Polygon, 22 Dec. 2016, www.polygon.com/2016/12/22/14046204/watch-dogs-2-race. Accessed 23 Jan. 2020.

Ferguson, Andrew Guthrie. The Rise of Big Data Policing: Surveillance, Race, and the Future of Law Enforcement. NYU P, 2017.

Frankenberg, Ruth. White Women, Race Matters: The Social Construction of Whiteness. U of Minnesota $\mathrm{P}, 1993$.

Fussell, Sidney. "Review: Watch Dogs 2 Makes Light Work of Being Watched." Rolling Stone, 15 Nov. 2016, www.rollingstone.com/glixel/reviews/review-watch-dogs-2-makes-light-work-of-beingwatched-w450539. Accessed 13 Sept. 2017.

Greenwald, Glenn. No Place to Hide: Edward Snowden, the NSA, and the U.S. Surveillance State. Hamish Hamilton, 2014.

Hennig, Martin. “Watch Dogs und die Heterotopie der Überwachung: Motive, Strukturen und Funktionen überwachter Welten in Digitalen Spielen." Überwachung und Kontrolle im Computerspiel, special issue of Paidia, edited by Martin Hennig and Marcel Schellong, 25 June 2020, www.paidia.de/heterotopie-der-ueberwachung. Accessed 15 July 2020.

Imtiaz, Khurram. "Watch Dogs Random Character Profiler Is often Hilarious, even Awkward." Gearnuke, 27 May 2014, www.gearnuke.com/watch-dogs-random-character-profiler-can-resultpretty-hilarious-descriptions/. Accessed 15 Jan. 2020.

Kamen, Matt. "Watch Dogs 2 is Masterful, Astute and finally Lives up to the Original's Promise." Wired, 24 Oct. 2016, www.wired.co.uk/article/watch-dogs-2-hands-on. Accessed 23 Jan. 2020.

Keogh, Brendan. A Play of Bodies: How We Perceive Videogames. MIT P, 2018.

Leonard, David J. "Virtual Anti-Racism: Pleasure, Catharsis, and Hope in Mafia III and Watch Dogs 2." Humanity \& Society, vol. 44, no. 1, 2020, pp. 111-30.

Lucas, Gerald R. "Speculative Fiction." The Encyclopedia of Twentieth-Century Fiction, vol. 2, edited by Brian W. Shaffer, Wiley, 2010, pp. 840-844. 
Marx, Gary T. Windows into the Soul: Surveillance and Society in an Age of High Technology. U of Chicago P, 2016.

Matsakis, Louise. "Scraping the Web Is a Powerful Tool. Clearview AI Abused It." Wired, 25 Jan. 2020, www.wired.com/story/clearview-ai-scraping-web/. Accessed 30 Jan. 2020.

McKeand, Kirk. "Watch Dogs 2 Review: Much Improved Hacker Sequel Makes a Smart Statement." Telegraph, 14 Nov. 2016, www.telegraph.co.uk/gaming/what-to-play/watch-dogs-2-review--much-improved-hacker-sequel-makes-a-smart/. Accessed 23 Jan. 2020.

Mulvey, Laura. "Visual Pleasure and Narrative Cinema." Screen, vol. 16, no. 3, 1975, pp. 6-18.

Murray, Soraya. On Video Games: The Visual Politics of Race, Gender and Space. I. B. Tauris, 2018.

Nakamura, Lisa. “Afterword: Racism, Sexism, and Gaming's Cruel Optimism.” Gaming Representation: Race, Gender, and Sexuality in Video Games, edited by Jennifer Malkowski and TreaAndrea M. Russworm. Indiana UP, 2017, pp. 245-50.

Ng, Jenna, and Jamie Macdonald. “'We Are Data': The Cyberpunk Imaginary of Data Worlds in Watch Dogs." cyberpunk and Visual Culture, edited by Graham J. Murphy and Lars Schmeink. Routledge, 2018, pp. 174-189.

O'Neil, Cathy. Weapons of Math Destruction: How Big Data Increases Inequality and Threatens Democracy. Crown, 2016.

Pasquale, Frank. The Black Box Society: The Secret Algorithms That Control Money and Information. Harvard UP, 2015.

Russworm, TreaAndrea M. "A Call to Action for Video Game Studies in an Age of Reanimated White Supremacy." Velvet Light Trap, no. 81, 2018, pp. 73-76.

Sinha, Ravi. "Watch Dogs Animation Director: "You'll Never See the Same Thing Twice Guaranteed." GamingBolt, 16 Apr. 2014, www.gamingbolt.com/watch-dogs-animation-directoryoull-never-see-the-same-thing-twice-guaranteed. Accessed 15 Jan. 2020.

“Toni Morrison.” Charlie Rose, 19 Jan. 1998, www.charlierose.com/videos/17664. Accessed 3 Mar. 2021.

Watch Dogs. Ubisoft, 2014.

Watch Dogs 2. Ubisoft, 2016.

Whitson, Jennifer R., and Bart Simon. "Game Studies meets Surveillance Studies at the Edge of Digital Culture: An Introduction to a Special Issue on Surveillance, Games and Play." Surveillance and Society, vol. 12, no. 3, 2014, pp. 309-19.

Zuboff, Shoshana. The Age of Surveillance Capitalism: The Fight for a Human Future at the New Frontier of Power. Public Affairs, 2019.

\section{NOTES}

1. Watch Dogs is spelled out whenever I refer to the series at large.

2. Throughout this article, I consistently use the one-word spelling videogame to highlight the specificity of the medium and not treat it as predominately a subcategory of games, even though the two overlap in some respects (cf. Keogh 12, n2). I occasionally use the term game, however, as a popular synonym for videogames whenever beneficial to the flow of reading. AAA commonly refers to videogames developed and marketed with top-tier budgets. 
3. Non-player character.

4. The clip features a montage of scenes from WD where the player purposefully kills NPCs with certain, assumedly deviant, markers in their profiles.

5. Toni Morrison famously described and conceptualized the dominance of the white gaze and the struggle to write against it in an interview with Charlie Rose ("Toni Morrison").

6. It is apparent that gender intersects with race and other markers of identity in myriad ways in these surveillance regimes. Although WD2 displays a more inclusive and more complex representation of gendered identities, the Watch Dogs series generally is defined by a male gaze because of its protagonists and other narrative and visual design choices. While unfortunately outside the scope of this article, an intersectional analysis of the franchise that complements the question of race with the questions of gender and other categories of identity is highly desirable, especially given the distinct histories of the surveillance of women, especially women of color, within and beyond the parameters sketched out in this article.

7. Recent news stories about a New York-based start-up called Clearview AI, which allegedly has developed a facial recognition tool for law enforcement that uses billions of images secretly gathered from social media platforms, indicate that such databases are steadily becoming more comprehensive (Matsakis). Nonetheless, the racial imbalance in current real-world applications of such technologies remains, at least for the time being.

8. Players can, however, place a false APB (all-points bulletin) on NPCs in WD2 by hacking them, which puts the focus of law enforcement on them and may lead to the in-game police's pursuit of that NPC.

9. For some reviews highlighting the racial politics and diversity of WD2, see D, Fussell, Kamen, and McKeand.

10. One notable exception is the seamless multiplayer mode "Online Invasion," which allows players to enter other players' singleplayer sessions. Even here, power is not distributed equally: in the PlayStation 4 version of WD2, everyone can be invaded but only paying subscribers of the PlayStation Plus service can hack others.

\section{ABSTRACTS}

This article reads Ubisoft's Watch Dogs, a series of open-world videogames, through and against the entangled histories of race and surveillance in the United States. Drawing especially on recent research at the intersections of STS (science and technology studies) and CRT (critical race theory), several aspects of the videogames' fictional world, game mechanics, plot, and visual and procedural representation are scrutinized. The first two Watch Dogs titles, I argue, both erase the realities of racializing surveillance in their conceptualization and simulation of a contemporary American surveillance society and prominently feature characters who embody the painful histories and the enduring present of racializing surveillance in the United States in several ways. These two opposing representations ultimately reproduce the racializing logics of contemporary digital surveillance as well as its lineage in American history in the ways that both whiteness and Blackness organize the operation of surveillance in the Watch Dogs franchise. 
INDEX

Keywords: surveillance, race, video games, Watch Dogs, Ubisoft, whiteness, Blackness, biometrics, predictive policing

\section{AUTHOR}

\section{SÖREN SCHOPPMEIER}

Sören Schoppmeier recently completed his Ph.D. thesis, titled "Playing American: Open-World Videogames, Ambient Operations, and the Reproduction of American Culture," at the John F. Kennedy Institute for North American Studies at Freie Universität Berlin. His published and forthcoming academic writing has dealt with a variety of videogames and franchises, including Grand Theft Auto, Watch Dogs, Red Dead Redemption, Far Cry 5, and Pony Island. 